Journal of Case Reports 2018;8(2):98-101

\title{
Pleomorphic Adenoma of Submandibular gland
}

\author{
Shouptik Basu' ${ }^{1}$, Trilokesh Patra ${ }^{2}$ \\ Departments of ${ }^{1}$ General Surgery, ${ }^{2}$ Radio-diagnosis; Bankura Sammilani Medical College, Bankura, West Bengal, India.
}

\section{Corresponding Author: \\ Dr. Shouptik Basu}

Email: dr.shouptik@gmail.com

This is an Open Access article distributed under the terms of the Creative Commons Attribution License (creativecommons.org/ licenses/by/3.0).

Received : December 27, 2017

Accepted : March 14, 2018

Published : April 15, 2018

\begin{abstract}
Background: Pleomorphic adenoma is the most commonly occurring tumor of the parotid gland $(80-90 \%)$. Submandibular and sublingual pleomorphic adenomas are quite uncommon constituting approximately $8-10 \%$ and are mostly malignant. A complete surgical excision of the tumor is the treatment of choice. Case Report: We report a case of a pleomorphic adenoma of the sub-mandibular gland in an adult male, its diagnosis and surgical management at a tertiary care teaching institute in Eastern India. Conclusion: Neck swellings are difficult to diagnose due to the fact that a myriad of clinical entities may have a common clinical picture. Upon clinical suspicion a careful pre-operative work up including imaging and fine needle aspiration cytology is advocated to arrive at a diagnosis.
\end{abstract}

Keywords: : Fine-Needle Biopsy, Mixed Tumor, Pleomorphic Adenoma, Submandibular Gland.

\section{Introduction}

Salivary gland tumors are relatively uncommon and constitute less than $2 \%$ of all head and neck tumors. Tumors from minor salivary glands have a higher risk for malignancy. Pleomorphic adenoma has a heterogeneous histology and has possibility of malignant transformation. Recurrences occur because of an incomplete capsule and presence of pseudopods. A complete surgical excision of the tumor is desirable [1]. This paper describes a case of pleomorphic adenoma involving the submandibular gland of an adult male and correlates the clinical, radiological and pathological findings to establish a diagnosis.

\section{Case Report}

A 43-year-old male presented with a gradual onset insidious swelling near the angle of the right mandible since past four years. The swelling has gradually increased over the years and attained its present size [Fig.1]. There was no history of pain, fever, loss of weight, excessive drooling of saliva, facial disfigurement, excessive drooling or difficulty in swallowing or chewing. The patient is a known smoker with no significant history of medical and surgical ailments. Local examination revealed three $\mathrm{cm}$ globular swelling not fixed to the skin but fixed to the deeper structures, located $1 \mathrm{~cm}$ below the angle of the mandible. The swelling was firm, non-compressible with distinct margins, having negative fluctuation and trans-illuminance tests. The swelling was palpable bi-manually during an oral examination with good dental hygiene.

The patient was evaluated with an initial ultrasound of the neck that revealed a $2.84 \times 2.01 \mathrm{~cm}$ well defined predominantly hypoechoic lesion with lobulated border and posterior acoustic enhancement [Fig.2]. Transverse power Doppler ultrasound showed profuse intralesional vascularity (mainly venous on Colour Doppler). Doppler indices - resistance index $<0.8$, pulsatality index $<2.0$ were suggestive of low resistance vessels, thus raising the suspicion of a soft tissue tumor likely a benign mixed 
tumor. A contrast CT scan revealed an ovoid inhomogeneously enhancing mass over the level IB and II of the neck [Fig.3,4]. Routine blood investigations were within normal limits. A ultrasound guided fine needle aspiration from the swelling revealed chondromyxoid stroma, normal to round cells with bland nuclei, basophilic cytoplasm spread singly, in small clusters and also admixed with the stromal elements raising the suspicion of a pleomorphic adenoma of the submandibular gland. Based on all the above findings a provisional diagnosis of pleomorphic adenoma was established and the patient was taken up for surgery.

After induction of general anaesthesia and skin preparation, positioning with chin rotated to the opposite side, with moderate neck extension a horizontal incision was made at least 2 finger breadths $(3-4 \mathrm{cms})$ below the mandible. The incision was carried down through the platysma to expose the anterior border of the sternocleidomastoid muscle posteriorly and the anterior belly of the digastric muscle. The posterior inferior aspect of the submandibular gland was identified immediately anterior to the sternocleidomastoid muscle where it overlays the posterior belly of the digastric muscle. Marginal mandibular nerve was preserved. The gland was mobilized and the tumor was excised preserving the lingual nerve and the facial artery [Fig.5]. The tumor was sent for histopathological examination. After thorough wound lavage and haemostasis a closed suction drain was put and the wound was closed in layers. The patient's postoperative phase was uneventful and the closed suction drain was removed after three days and the skin sutures were removed after a week. Antibiotics were used as per the routine hospital policy.

The excised specimen was globular and had a nodular surface approximately $3 \mathrm{~cm}$ in diameter. On transverse section it was variegated mainly smooth, whitish with small blackish grey areas. Histopathology showed a biphasic appearance

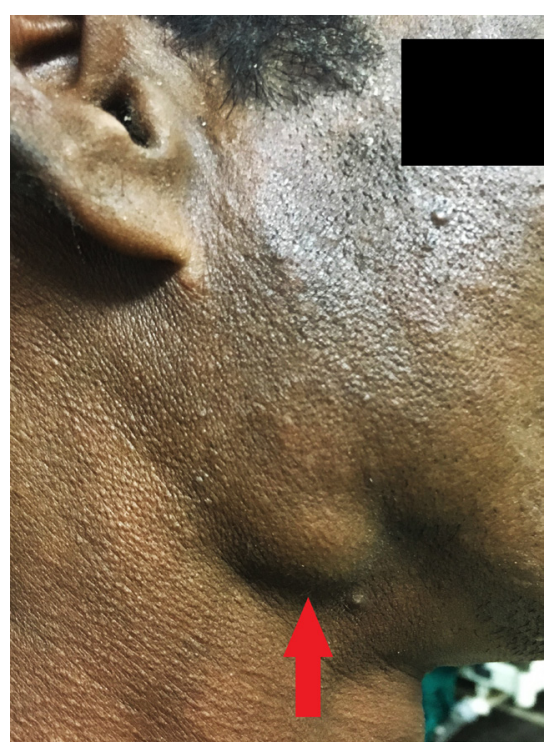

Fig.1: Profile view of the patient showing the submandibular swelling (red arrow).

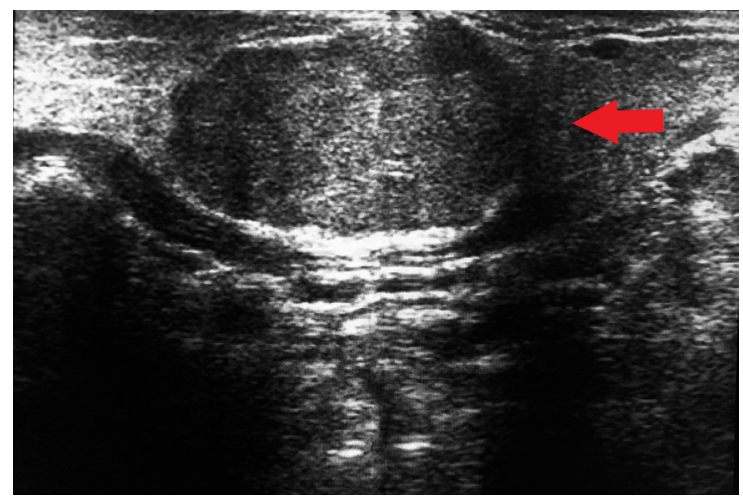

Fig.2: Ultrasound shows $2.84 \times 2.01 \mathrm{~cm}$ well defined predominantly hypoechoic lesion with lobulated border and posterior acoustic enhancement (arrow-swelling).

resulting from intimate admixture of epithelial and stroma. Epithelial elements were glandular in nature and the neoplastic glands had basally located cells and cuboidal to flattened myoepithelial cells. The stroma had a non-specific chondromyxoid appearance, confirming the diagnosis of a pleomorphic adenoma of the submandibular gland [Fig.6,7]. The patient is currently doing well and has attended the clinic for follow up. There have been no recurrences. 

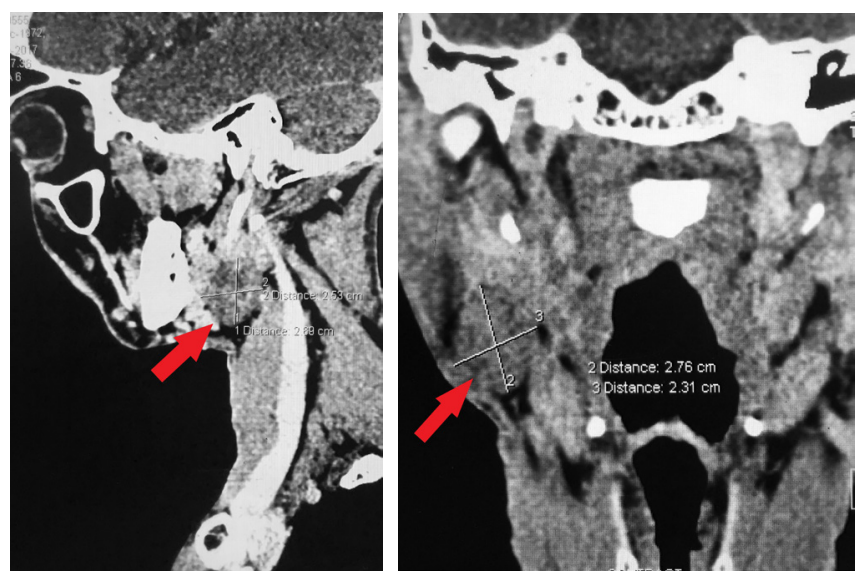

Fig.3,4: Saggital and coronal contrast $C T$ scan revealed an ovoid in-homogeneously enhancing mass over the level IB and II of the neck (arrow-swelling).

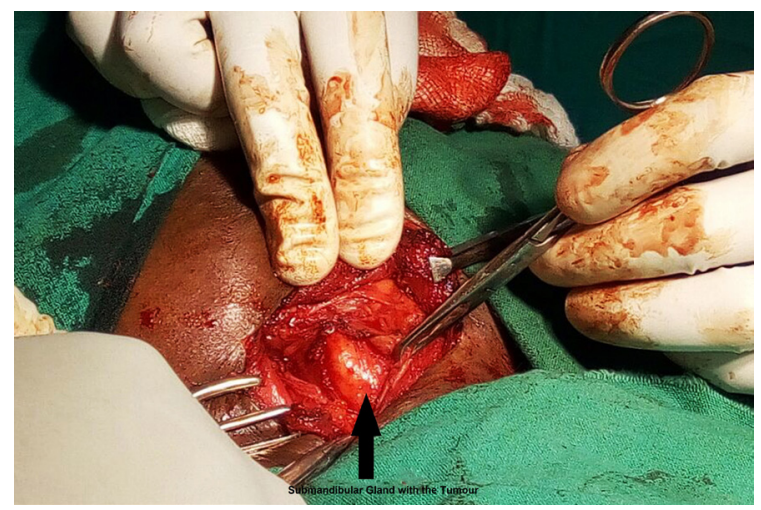

Fig.5: Intra-operative photograph of the swelling being excised (swelling-black arrow).

\section{Discussion}

Previous studies in the past state that submandibular pleomorphic adenomas present as a painless swelling in the submandibular region, with a slight female preponderance as compared to males, typically arising between the $3^{\text {rd }}$ to the $5^{\text {th }}$ decades [2-5]. Here in our case the patient presented with an insidious onset firm swelling for the past four years that raised clinical suspicion. Our decision to evaluate the swelling with an initial ultrasound proved fruitful. The ultrasound picture was in favour of a benign mixed tumor because of its characteristic lobulated appearance and posterior enhancement. The ultrasound and pre-operative

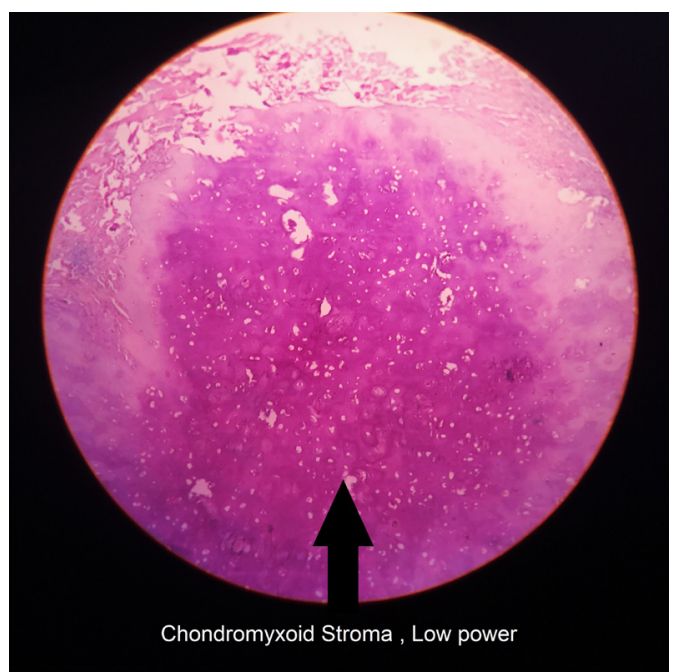

Fig.6: Chondromyxoid stroma (low power-black).

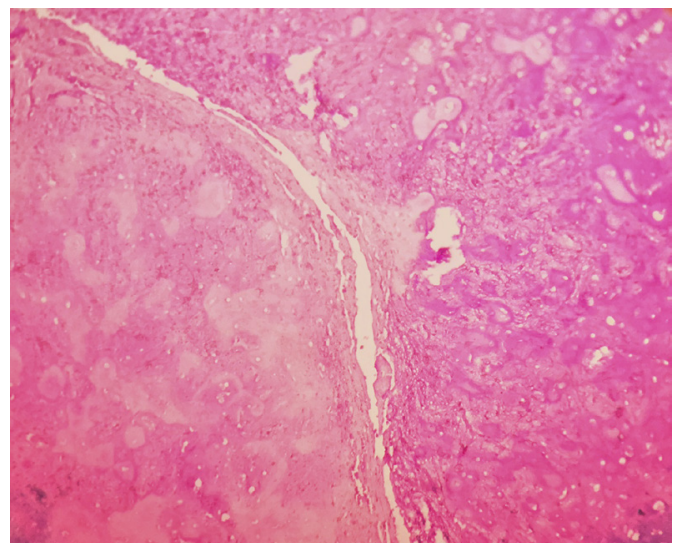

Fig.7: Junctional zone between epithelial (right) and stromal elements (left) in low power.

assessment with Fine Needle Aspiration Cytology (FNAC) helps to differentiate between tumors and inflammatory conditions or enlarged lymph nodes [6-8]. A needle no more than $18 \mathrm{G}$ should be used to prevent undue seeding of tumor cells in the adjoining regions. Pleomorphic adenomas are known to recur because of their classical pseudopods like excrescences. A CT scan aids in the diagnosis and thus helps to review the anatomical relation to the adjoining structures. Magnetic Resonance Imaging (MRI) helps to determine perineural invasion and soft tissue involvement but is only comparable to a CT scan [1]. It lacks the specificity to differentiate between a benign and 
malignant lesion. Hence a MRI wasn't considered in this case. Surgery is the only recommended mode of treatment for submandibular pleomorphic adenomas. We planned for surgery and decided to go for an excision biopsy. The most common complication arising from surgery is a temporary marginal mandibular nerve neurapraxia in $25 \%$ of cases that can be managed conservatively, and takes approximately three months to resolve [9]. Thus the incision for surgery must be carefully planned at-least 3-4 cms below the lower end of the mandible to avoid nerve injury. Histopathological studies concluded that the swelling originated from the submandibular gland because of its classical architecture of being a mixed gland comprising of both serous and mucous glands. The essential components are the capsule, epithelial and myoepithelial cells and mesenchymal or stromal elements. It is essential to follow up such patients undergoing an excision of such tumors because such tumors are known to recur, however the incidence for the submandibular gland is less. Pleomorphic adenomas may also undergo malignant changes. Our patient had to be followed up regularly and repeat ultrasounds and CT scans have not shown any recurrences. Recurrences and overt malignancy are treated with a modified radical neck dissection and post-operative radiotherapy [1] .

\section{Conclusion}

The head and neck is a complex region. A multitude of different clinical entities may present in the same location and obscure a diagnosis. Submandibular pleomorphic adenomas are relatively rare. We recommend an integrated approach with clinical, radiological and cytological studies to elicit the nature of the swelling. Since the incidence of a submandibular swelling being malignant is high it is crucial to plan the surgery and evaluate the relation of the swelling to the surrounding structures. We also recommend a post-operative follow-up of the patient because such tumors do recur, and they may require a radical surgery later, even though many literature reviews state that the incidence is quite rare.

Acknowledgement: Department of Pathology, Bankura Sammilani Medical College and Hospital for providing the slides from the resected specimen, for the histopathological pictures.

Contributors: SB: drafting of manuscript; TP: critical inputs in manuscript and imaging. SB will act as guarantor. Both authors approved the final version of this manuscript.

Funding: None; Competing interests: None stated.

\section{References}

1. Brunicardi F Charles, Andersen Dana K, Billiar Timothy R, Dunn David L, Hunter John G. Schwartz's Principles of Surgery. Tenth edition. New York: McGraw-Hill Education. pp. 599.

2. Munir N, Bradley PJ. Pleomorphic adenoma of the submandibular gland: An evolving change in practice following review of a personal case series. Eur Arch Otorhinolaryngol. 2007;264:1447-1452.

3. de Oliveira FA, Duarte EC, Taveira CT, Máximo AA, de Aquino EC, Alencar Rde C, et al. Salivary gland tumor: A review of 599 cases in a Brazilian population. Head Neck Pathol. 2009;3:271-275.

4. Alves FA, Perez DE, Almeida OP, Lopes MA, Kowalski LP. Pleomorphic adenoma of the submandibular gland: Clinicopathological and immunohistochemical features of 60 cases in Brazil. Arch Otolaryngol Head Neck Surg. 2002;128:1400-1403.

5. Eveson JW, Cawson RA. Salivary gland tumors. A review of 2410 cases with particular reference to histological types, site, age and sex distribution. J Pathol. 1985;146:51-58.

6. Spiro RH. Salivary neoplasms: Overview of a 35year experience with 2,807 patients. Head Neck Surg. 1986;8:177-184.

7. Laskawi R, Ellies M, Arglebe C, Schott A. Surgical management of benign tumors of the submandibular gland: a follow-up study. J Oral Maxillofac Surg 1995;53:506-508.

8. Ashraf A, Shaikh AS, Kamal F, Sarfraz R, Bukhari MH. Diagnostic reliability of FNAC for salivary gland swellings: A comparative study. Diagn Cytopathol. 2010;38:499-504.

9. Kennedy PJ, Poole AG. Excision of the submandibular gland: minimizing the risk of nerve damage. Aust N Z J Surg. 1989;59:411-414. 